\title{
Mucosal and transdermal vaccine delivery strategies against COVID-19
}

\author{
Ashwini Kumar ${ }^{1}$. Awanish Kumar ${ }^{1} \mathbb{C}$ \\ Accepted: 5 May 2021 / Published online: 15 May 2021 \\ (c) Controlled Release Society 2021
}

\begin{abstract}
The years 2020 and 2021 have witnessed a COVID-19 pandemic caused by the SARS-CoV-2 virus. However, these years have also witnessed certain remarkable scientific achievements. Researchers across the globe have been trying extremely hard and accomplished in bringing vaccines a great variety of COVID-19 vaccines. Though the route of administration for the majority of these vaccines has been the intramuscular route (invasive), some laboratories are developing formulations intended for transmucosal and transcutaneous (non-invasive) administration, which are in the early phases of pre-clinical and clinical development. This short report discusses these unconventional formulations against COVID-19, in brief, to stress the importance of research in the field of drug delivery.
\end{abstract}

Keywords COVID-19 $\cdot$ Vaccine $\cdot$ Delivery $\cdot$ Alternative approach $\cdot$ Non-invasive

\section{Technical note}

As of April 28, 2021, the total official number of COVID19 cases worldwide has crossed the 147 million mark with more than 3.1 million deaths and 192 affected countries as per Johns Hopkins Coronavirus Resource Web site [1]. The research community of the entire world is working very hard to develop COVID-19 vaccines at an unprecedented pace. As per a recent report published on the Web site of the World Health Organization (WHO), 87 vaccine candidates are in various clinical development phases while 186 vaccine candidates are in different phases of pre-clinical development [2]. Most of the COVID-19 vaccine (either in the developmental stage or a few developed) will be administered through the conventional mode. The conventional mode of vaccine administration requires intramuscular injection using a hypodermic needle while the conventional vaccine development platform uses attenuated microorganisms, killed microorganisms, protein subunit vaccine, and conjugated vaccine. Certain novel vaccine platforms utilize virus-like particles, DNA, and mRNA of the concerned microorganism. Various vaccines are currently in phase $3 / 4$ trial and are approved for administration with conditions

Awanish Kumar

drawanishkr@gmail.com; awanik.bt@ nitrr.ac.in

1 Department of Biotechnology, National Institute of Technology, Raipur, CG, India mentioned in Table 1 [2-5]. The vaccines mentioned in Table 1 are administered intramuscularly. The majority of other vaccines that are in various phases of clinical and pre-clinical development are also administered through the intramuscular route.

Since SARS-CoV-2 (the virus that causes COVID19) primarily affects the lungs; therefore, the generation of mucosal immunity is crucial for protection. Eliciting mucosal immunity would result in enhanced production of effective specific IgA at the mucosal site, neutralizing $\mathrm{IgG}$, and specific T-cell response. Generation of tissue-resident memory, T-cells in lungs, and nasal epithelia is critical to mitigating a secondary infection in the host. The local mucosal immune response is important in this case to mitigate the replication of the SARS-CoV-2 virus in nasal epithelia. Therefore, research on the importance of mucosal immunity is gaining momentum and it has resulted in the research on specific adjuvants and antigens that can result in homing of parenterally primed immune cells to the mucosal sites as well. However, this mechanism has perhaps not resulted in better homing of these primed cells to the lungs. Thus, the nasal route is still considered more suitable for direct action in case of respiratory infection like COVID-19 $[6,7]$. But the mucosal administration is still not considered the usual route for immunization. However, certain research groups and pharmaceutical companies have come up with mucosal formulations of the COVID-19 vaccine. Most of the investigated mucosal vaccines for COVID-19 are being 
Table 1 Vaccines currently approved or approved with emergency use authorization and their clinical development phase

\begin{tabular}{|c|c|c|c|c|}
\hline S. no & Vaccine name & Company & Technology platform & $\begin{array}{l}\text { Clini- } \\
\text { cal } \\
\text { phase }\end{array}$ \\
\hline 1. & Comirnaty or BNT162b2 & Pfizer-BioNTech, USA & mRNA in lipid nanoparticle & 4 \\
\hline 2. & mRNA-1273 & Moderna, USA & mRNA in lipid nanoparticle & 4 \\
\hline 3. & Covishield or AZD1222 & AstraZeneca and University of Oxford, UK & $\begin{array}{l}\text { Chimpanzee adenovirus-based vector containing } \\
\text { gene for S-protein (non-replicating) }\end{array}$ & 4 \\
\hline 4. & Covaxin or BBV152 & Bharat Biotech, India & Inactivated virus & 3 \\
\hline 5. & Ad26.COV2.S & Janssen/Johnson \& Johnson, USA & $\begin{array}{l}\text { Recombinant non-replicating adenovirus } 26 \text { contain- } \\
\text { ing DNA for spike protein }\end{array}$ & 3 \\
\hline 6. & Sputnik V & Gamaleya Research Institute, Russia & $\begin{array}{l}\text { Recombinant non-replicating adenovirus } 26 \text { and } \\
\text { adenovirus } 5 \text { vector containing DNA for spike } \\
\text { protein }\end{array}$ & 3 \\
\hline 7. & CoronaVac & Sinovac, China & Inactivated virus & 4 \\
\hline 8. & BBIBP-CorV & Beijing Institute of Biological Products, China & Inactivated virus & 3 \\
\hline 9. & Convidicea & CanSino Biologics, China & Recombinant adenovirus type 5 virus & 3 \\
\hline
\end{tabular}

developed for the intranasal route. Besides the intranasal mucosal routes, tablet and capsule-based COVID-19 vaccines are also being investigated which gives us a hope of moving to a non-conventional route of vaccination that could be non-invasive. A third minimally invasive technique that also holds a lot of potential for eliciting significant immune response is vaccine administration using a microneedle. All these innovative investigations for the development of the non-conventional COVID-19 vaccine are discussed below.

\section{Intranasal vaccines}

The most promising intranasal COVID-19 vaccine candidates are COVI-VAC (live attenuated) from Codagenix (USA) and Serum Institute (India), replicating viral vector-based RBD expressing vaccine being developed by the University of Hong Kong and Beijing Wantai Biological Pharmacy (China). CIGB-669 by Centre for Genetic Engineering and Biotechnology (Cuba), AdCOVID from collaborative work between Altimmune Inc. (USA) and the University of Alabama (USA); Razi Cov Pars by Razi Vaccine and Serum Research Institute (Iran), and BBV154 from Bharat Biotech India (licensed from Washington University, School of Medicine in St. Louis, USA) [2]. In a recent development, COVI-VAC (a single-dose intranasal live-attenuated vaccine) has proceeded to phase 1 trial [8]. As per the information available from the Codagenix Web site, the COVI-VAC protects against all the proteins and not just the spike protein of SARS-CoV-2 which can most likely be beneficial against a range of SARS-CoV-2 strains [9]. The AdCOVID candidate from Altimmune (USA) and the University of Alabama (USA) is a replication-deficient human adenovirus 5 (hAd5) vectored single-dose intranasal vaccine encoding the receptor-binding domain of the spike protein of SARS-CoV-2 activating both mucosal and systemic arm of immunity. The latest pre-print publication states that AdCOVID elicited strong serum neutralizing antibodies, $\mathrm{T}$-cell response $\left(\mathrm{CD} 4^{+}\right.$and $\mathrm{CD}^{+}$response with a Th- 1 like a cytokine expression), and mucosal IgA in the respiratory tract. AdCOVID is reported to be stable over several months at room temperature. The information available from the clinical trial Web site reports that the phase 1 clinical trial of AdCOVID has commenced, and the study is presently recruiting subjects $[10,11]$. Washington University, School of Medicine, St. Louis (USA), is developing an intranasal non-replicating chimpanzee adenovirus vectored vaccine expressing the spike protein gene of SARS-CoV-2, and it has entered the phase of licensing agreement with Bharat Biotech India to manufacture the candidate (name BBV154). The BBV154 candidate has entered phase 1 clinical trial [12]. Recently, a research team has shown that a single intranasal dose elicited significant neutralizing antibodies and T-cell response against the SARS-CoV-2 virus on post 1-month challenge after the day of immunization [13]. In another development toward designing a potential intranasal vaccine against SARS-CoV-2, Meissa vaccine (USA) has designed a vaccine candidate which is based on codon optimization technique and currently in the pre-clinical GMP manufacturing stage using AttenuBlock ${ }^{\mathrm{TM}}$ proprietary technology [14]. The company has used the same technology for the development of the respiratory syncytial virus intranasal vaccine candidate which is currently in phase 2 trial [15]. Utrecht University Netherlands, Wageningen Bioveterinary Research University Netherlands, and Intravacc Netherlands are developing an intranasal vaccine against SARS-CoV-2 using reverse genetics technology with Newcastle Disease Virus as the vector for expressing the spike protein of SARSCoV-2 [16]. Intravacc Netherlands is also developing a nasal 
spray vaccine consisting of its proprietary outer membrane vesicle (OMV) click technology where the OMV is coupled with the recombinant spike protein of SARS-CoV-2. The company recently announced positive results in pre-clinical studies in mice and hamsters where the vaccine candidate elicited significant mucosal and systemic immunity [17]. Shifting from viral vectors, an interesting intranasal vaccine candidate is also being investigated (pre-clinical stage) by BlueWillow Biologics, USA, and Medigen Vaccine Biologics Corporation, Taiwan. The partnership is utilizing an oilin-water nanoemulsion vehicle (400-500 nm size) adjuvant named NanoVax ${ }^{\circledR}$ platform from BlueWillow to deliver the SARS-Cov-2 S-2P ectodomain (that maintains the prefusion spike confirmation) that elicit a strong systemic and mucosal immune response with mucosal interleukin-17 stimulation $[18,19]$. The vaccine is currently named S-2P-NE-01 that has shown a significant IgA response both in serum and bronchoalveolar lavage. The consolidated information of these intranasal vaccines discussed above is provided in Table 2.

\section{Oral route}

Interestingly, two companies namely Vaxart Inc. (USA) and iosBio Pharma (UK) are developing non-conventional oral formulations for the SARS-CoV-2 vaccines. Vaxart [using its patented VAAST ${ }^{\mathrm{TM}}$ (Vector-Adjuvant-Antigen Standardized Technology) platform] is developing a tablet formulation of non-replicating adenovirus type 5 (Ad5)-based COVID-19 vaccine. The tablet formulation would cover various issues faced by a parenteral formulation such as cold storage distribution, requirement of a healthcare worker for administration, and more importantly the pain associated with the administration which is a major patient compliance issue. On the immunity front, the vaccine candidate has been reported to elicit a very high titer of neutralizing antibodies in lungs, a dose-dependent increase in IgG levels, and generation of antigen-dependent $\mathrm{CD} 4^{+}$and $\mathrm{CD} 8^{+}$cells in animal models [20]. The latest press release from the Vaxart Web site (dated February 3,2021) says that the candidate reached the primary and secondary endpoint of safety and immunogenicity respectively in the phase 1 clinical trial (clinicaltrials. gov; Identifier: NCT04563702) [21]. The tablet form of the vaccine induces a significant $\mathrm{CD} 8^{+} \mathrm{T}$-cell response, and it was also found to be substantially protective against the new strains of the SARS-CoV-2. The vaccine candidate (named VXA-CoV2-1) is using both the outer spike protein (S) and the nucleocapsid protein $(\mathrm{N})$. The $\mathrm{N}$ protein is more conserved and thus less prone to mutation. Therefore, it could potentially provide immunity to different strains. Secondly, $\mathrm{N}$ protein is said to be a better target of T-cell response. The press release shows that a total of 495 subjects have been administered the vaccine candidate with no report of any serious adverse effect. The primary immune responses that were observed, as per the official press release, are $\mathrm{CD} 8^{+}$ cytotoxic T-cell responses against $\mathrm{S}$ and $\mathrm{N}$ antigens (responsible for long-lasting cross-reactive immunity). An elevation in plasma B-cells and an upregulation of mucosal homing

Table 2 Detailed of COVID-19 vaccine candidates designed for alternative delivery

\begin{tabular}{|c|c|c|c|c|c|}
\hline S. no & Vaccine candidate name & Company & Feature & Delivery route & Clinical phase \\
\hline 1. & CoviVac & $\begin{array}{l}\text { Codagenix USA and Serum } \\
\text { Institute, India }\end{array}$ & Live attenuated & Intranasal & 1 \\
\hline 2. & CIGB-669 (Mambisa) & $\begin{array}{l}\text { Centre for Genetic Engineer- } \\
\text { ing and Biotechnology, } \\
\text { Cuba }\end{array}$ & Protein subunit & Intranasal & 2 \\
\hline 3. & BBV154 & $\begin{array}{l}\text { Washington University } \\
\text { School of Medicine in St. } \\
\text { Louis, USA and Bharat } \\
\text { Biotech, India }\end{array}$ & $\begin{array}{l}\text { ChAd viralvector expressing } \\
\text { spike protein }\end{array}$ & Intranasal & 1 \\
\hline 4. & AdCOVID & $\begin{array}{l}\text { Altimmune Inc. USA and } \\
\text { University of Alabama } \\
\text { Birmingham, USA }\end{array}$ & $\begin{array}{l}\text { Non-replicating Adenovirus } \\
\text { type } 5 \text { expressing the RBD } \\
\text { of SARS-CoV-2 spike } \\
\text { protein }\end{array}$ & Single dose Intranasal & Pre-clinical investigation \\
\hline 5. & Unnamed & $\begin{array}{l}\text { BlueWillow Biologics, USA } \\
\text { and Medigen Vaccine Bio- } \\
\text { logics Corporation, Taiwan }\end{array}$ & $\begin{array}{l}\text { Oil-in-water nanoemulsion as } \\
\text { carrier and adjuvant }\end{array}$ & Intranasal & Pre-clinical investigation \\
\hline 6. & VXA-CoV2-1 & Vaxart Inc., USA & $\begin{array}{l}\text { Non-replicating Adenovirus } \\
\text { type } 5 \text { containing } \mathrm{S} \text { and } \mathrm{N} \\
\text { proteins }\end{array}$ & Oral tablet & 1 \\
\hline 7. & OraPro-COVID-19 & $\begin{array}{l}\text { iosBio Pharma (earlier } \\
\text { known as Stabilitech Biop- } \\
\text { harma), UK }\end{array}$ & $\begin{array}{l}\text { Non-replicating Adenovirus } \\
\text { type } 5 \text { containing spike pro- } \\
\text { tein DNA of SARS-CoV-2 }\end{array}$ & Oral capsule & 1 \\
\hline
\end{tabular}


receptor on these cells further indicated B-cell activation, elevation in pro-inflammatory Th1 cytokine (crucial for an anti-viral response), and IgA response in all the subjects [21]. iosBio Pharma (UK) is developing an oral capsule-based COVID-19 vaccine using the OraPro ${ }^{\mathrm{TM}}$ platform. Recently, the iosBio Pharma collaborated with Therm-SB technology and ImmunityBio (US-based biopharmaceutical company) for developing a human Adenoviral (hAd5) vector-based COVID-19 vaccine. The vaccine candidate uses a modified spike protein gene (S-fusion) and nucleocapsid protein gene with an enhanced T-cell stimulation domain (N-ETSD) [22]. In a recent study, the team reported a protective antibody response and generated a Th1-dominant T-cell response after a subcutaneous and an oral boost that has shown the protection in Rhesus macaques monkey with SARS-CoV 2 challenge. Significant immunity was observed at both the lower and upper respiratory tract of the animals. Another striking feature of the hAd5 vector (as the delivery vehicle) is that it can be used even in the pre-formed immunity against adenoviruses [22]. This vaccine candidate has entered in phase 1 trial [ClinicalTrials.gov Identifier: NCT04732468; ClinicalTrials.gov Identifier: NCT04591717]. The company recently reported a pre-clinical study in mice where they demonstrated significant immunogenicity when the vaccine candidate was used intranasally [23].

\section{Microneedle: minimally invasive technique}

Microneedle patch is a modified transdermal patch where the surface of the patch (that comes in the contact with the skin) consists of countable optimized microscopic sharp projections that deliver $\operatorname{drug}(\mathrm{s})$ inside the skin where the patient experiencing minimal pain. The microneedles can be solid, hollow, swellable, or degradable. The hypodermic needle that is used in common syringes for drug administration is a cause of pain and fear of pain among the patients. Microneedle patches solve the problem of pain and other comorbidities while delivering the drugs. With vaccine administration, microneedle patches could prove more beneficial since the dermis layer has the presence of a multitude of antigenpresenting cells. Microneedle patches, unlike hypodermic syringes, are also effective in significantly reducing the risk of transmission of infection and release of sharp biohazard waste. It has also been reported that microneedle-based formulations may show less dependence on cold chain storage, therefore reducing the cost drastically. Another benefit of considering a microneedle formulation for vaccine delivery is the minimization of healthcare facilities and significantly reduced dependence on healthcare workers for vaccine administration, especially in low-income countries. Owing to the large population of active antigen-presenting cells in the epidermis and dermis together, the microneedle approach could potentially reduce the dose of vaccine thereby might reduce the overall vaccination cost. The dissolvable microneedle matrix encapsulated vaccine candidates are also reported to be stable for long period without the stringent requirement of cold chain storage [24, 25]. A recently published data on the investigation of microneedles for delivering SARS-CoV-2 antigen for COVID-19 vaccine is reported by Kim et al. [26]. Because of their previous experience with a similar study for the MERS virus, they could rapidly design the COVID-19 vaccine candidate and tested the vaccine encapsulated microneedle on mice model. The team used carboxymethyl cellulose as an encapsulating and dissolvable biopolymer. The report describes the substantial generation of vaccine-induced antibodies within 2 weeks of administration. Due to an early stage of the study, the team could not study and report regarding the generation of neutralizing antibodies [26]. The study provides a ray of hope for investigating the microneedle-based COVID-19 vaccine. In another study, Kuwentrai et al. [27] designed microneedle patches using low molecular weight hyaluronic acid that could deliver the RBD domain peptide of the SARS-CoV-2 spike protein. A study in the mouse model demonstrated the significant generation of antibody and T-cell response. However, commercial production remains a distant dream owing to the large-scale manufacturing and regulatory considerations. But ongoing research gives hope that we could develop various therapeutic and preventive modalities against this pandemic in near future.

\section{Discussion}

COVID-19 disease (caused by SARS-CoV-2) has become a pandemic and affecting people in the whole world. The vaccine is urgently needed for society to combat this pandemic. The conventional vaccine delivery system usually follows a parenteral route that is invasive and requires stringent cold chain storage. Novel vaccine delivery systems and noninvasive routes can solve these challenges. Currently, largescale vaccination is being done in various countries using few vaccines approved under the emergency usage approval category. However, since the intramuscular route has been the most common route for administering the vaccine, all these currently used COVID-19 vaccines are formulated to deliver through the intramuscular route. However, development of novel vaccine candidates and tweaking with nuances of drug delivery systems, researchers have designed novel vaccine candidates that have shown brilliant results in vitro, in pre-clinical, and in initial stages of clinical trials. Owing to the route of infection and nature of SARS-CoV-2, the mucosal route of vaccine administration is being propagated as a more robust and viable route for COVID-19 vaccine delivery. Most of the mucosal COVID-19 vaccine candidates being developed are designed and formulated to be 
administered intranasally, while two candidates have taken the oral route which is non-conventional for the vaccine.

Besides the intranasal and oral mucosal candidates, two reports on designing microneedle patches for COVID-19 vaccine delivery have also been discussed in this article. But as stated, commercial production remains a distant dream owing to various manufacturing and regulatory conditions. We, therefore, hope that successful translation of these mucosal candidates and could provide us much specific/better mucosal and systemic immunity in the fight against the SARS-CoV-2 virus, i.e., COVID-19 pandemic.

Acknowledgements The authors are thankful for the support and environment provided by the Department of Biotechnology, National Institute of Technology, Raipur (CG), India, for this manuscript.

Author contribution Both authors contributed equally to the manuscript including conception, drafting, and proofreading.

Availability of data and materials Discussed information/data derived from public resources and made available with the article.

\section{Declarations}

Ethics approval and consent to participate This manuscript is a reviewtype article and does not involve a research protocol requiring approval by the relevant institutional review board or ethics committee.

\section{Consent for publication Yes.}

Competing interests The authors declare no competing interests.

\section{References}

1. https://coronavirus.jhu.edu/map.html; Accessed on April 28, 2021.

2. https://www.who.int/publications/m/item/draft-landscape-ofcovid-19-candidate-vaccines; Accessed on February 27, 2021.

3. https://www.raps.org/news-and-articles/news-articles/2020/3/ covid-19-vaccine-tracker; Accessed on February 27, 2021.

4. Teijaro JR, Farber DL. COVID-19 vaccines: modes of immune activation and future challenges. Nat Rev Immunol. 2021.

5. https://www.gavi.org/vaccineswork/covid-19-vaccine-race; Accessed on February 27, 2021

6. Moreno-FierrosL, García-Silva I, Rosales-Mendoza S. Development of SARS-CoV-2 vaccines: should we focus on mucosal immunity? Expert Opinion Biol Ther. 2020;20(8):831-836.

7. Chung JY, Thone MN, Kwon YJ. COVID-19 vaccines: the status and perspectives in delivery points of view. Adv Drug Deliv Rev. 2021;170:1-25.

8. https://clinicaltrials.gov/ct2/show/NCT04619628?term= Codagenix \& cond $=$ COVID $-19 \& \mathrm{draw}=2 \& \mathrm{rank}=1$; Accessed on April 10, 2021.

9. https://codagenix.com/vaccine-programs/covid-19/; Accessed on March 1, 2021

10. King RG, Silva-Sanchez A, Peel JN et al. Single-dose intranasal administration of AdCOVID elicits systemic and mucosal immunity against SARS-CoV-2 in mice. bioRxiv 2020;10.10.331348.
11. https://clinicaltrials.gov/ct2/show/NCT04679909?term= AdCovid \&cond $=$ COVID-19\&draw $=2 \&$ rank $=1$; Accessed on April 28, 2021

12. https://clinicaltrials.gov/ct2/show/NCT04751682; Accessed on April 28, 2021.

13. Hassan AO, Feldmann F, Zhao H, Curiel DT, Okumura A et al. A single intranasal dose of chimpanzee adenovirus-vectored vaccine protects against SARS-CoV-2 infection in rhesus macaques. Cell Rep Med. 2021: 100230. https://doi.org/10.1016/j.xcrm.2021. 100230.

14. https://www.meissavaccines.com/technology ; Accessed on April $28,2021$.

15. https://www.meissavaccines.com/post/meissa-announces-1st-dosingin-phase-2-study-of-intranasal-live-attenuated-vaccine-candidate-forrsv; Accessed on April 28, 2021.

16. https://www.wur.nl/en/Research-Results/Research-Institutes/ Bioveterinary-Research/show-bvr/Development-of-an-intranasalvaccine-against-COVID-19.htm; Accessed on March 3, 2021.

17. https://www.intravacc.nl/news/intravacc-announces-positive-preclinical-data-intranasal-sars-cov-2-candidate-vaccine/; Accessed on April 28, 2021.

18. https://bluewillow.com/medigen-and-bluewillow-biologics-partnerto-develop-intranasal-vaccine-for-sars-cov-2/ ; Accessed on April 28,2021

19. http://www.medigenvac.com/public/en/news/detail/61?from sort=2; Accessed on April 28, 2021.

20. Moore AC, Dora EG, Peinovich Net al. Pre-clinical studies of a recombinant adenoviral mucosal vaccine to prevent SARS-CoV-2 infection.bioRxiv 2020.09.04.283853.

21. https://investors.vaxart.com/news-releases/news-release-details/ vaxart-announces-positive-preliminary-data-phase-1-clinical; accessed on April 28, 2021.

22. Gabitzsch E, Safrit JT, Verma M, Rice A, Seiling P et al. Complete protection of nasal and lung airways against SARS-CoV-2 challenge by antibody plus Th1 dominant $\mathrm{N}$ - and $\mathrm{S}$-specific T-cell responses to subcutaneous prime and thermally-stable oral boost bivalent hAd5 vaccination in an NHP study. bioRxiv; March 26, 2021. https://doi.org/10.1101/2020.12.08.416297.

23. Rice A, Verma M, Shin A, Zakin L, Sieling P, Tanaka S et al. The dual-antigen Ad5 COVID-19 vaccine delivered as an intranasal plus subcutaneous prime elicits Th1 dominant T-cell and humoral responses in CD-1 mice. bioRxiv; March 26, 2021; https://doi.org/ 10.1101/2021.03.22.436476.

24. Tran KTM, Gavitt TD, Farrell NJ et al. Transdermal microneedles for the programmable burst release of multiple vaccine payloads. Nat Biomed Eng. 2020. https://doi.org/10.1038/ s41551-020-00650-4.

25. O'Shea J, Prausnitz MR, Rouphael N. Dissolvable microneedle patches to enable increased access to vaccines against SARSCoV-2 and future pandemic outbreaks. Vaccines. 2021;9(4):320.

26. Kim E, Erdos G, Huang S, Kenniston TW, Balmert SC, Carey $\mathrm{CD}$, et al. Microneedle array delivered recombinant coronavirus vaccines: Immunogenicity and rapid translational development. EBioMedicine. 2020;55:102743.

27. Kuwentrai C, Yu J, Rong L, Zhang BZ, Hu YF, Gong HR, et al. Intradermal delivery of receptor-binding domain of SARS-CoV-2 spike protein with dissolvable microneedles to induce humoral and cellular responses in mice. Bioeng Transl Med. 2020;6(1):e10202.

Publisher's Note Springer Nature remains neutral with regard to jurisdictional claims in published maps and institutional affiliations. 